\title{
STRUCTURAL AND FUNCTIONAL DIVERSITY OF GLUTAREDOXINS IN YEAST
}

\author{
Enrique Herrero*, Gemma Bellí and Celia Casas \\ Departament de Ciències Mèdiques Bàsiques, IRB Lleida, Universitat de \\ Lleida, Montserrat Roig 2, 25008-Lleida, Spain
}

\footnotetext{
*Address correspondence to this author at the Departament de Ciències Mèdiques Bàsiques, IRBLleida, Universitat de Lleida, Montserrat Roig 2, 25008-Lleida, Spain. Phone: (34)973702409; Fax: (34)973702426; E-mail: enric.herrero@cmb.udl.cat
}

Running title: Structure and functions of glutaredoxins 


\begin{abstract}
Glutaredoxins are defined as thiol disulfide oxidoreductases that reduce disulfide bonds employing reduced glutathione as electron donor. They constitute a complex family of proteins with a diversity of enzymatic and functional properties. Thus, dithiol glutaredoxins are able to reduce disulfide bonds and deglutathionylate mixed disulfides between glutathione and cysteine protein residues. They could act regulating the redox state of sulfhydryl residues of specific proteins, while thioredoxins (another family of thiol disulfide oxidoreductases which employ NADPH as electron donor) would be the general sulfhydryl reductants. Some dithiol glutaredoxins such as human Grx2 form dimers bridged by one iron-sulfur cluster, which acts as a sensor of oxidative stress, therefore regulating the activity of the glutaredoxin. The ability to interact with iron-sulfur clusters as ligands is also characteristic of monothiol glutaredoxins with a CGFS-type active site. These do not display thiol oxidoreductase activity, but have roles in iron homeostasis. The three members of this subfamily in Saccharomyces cerevisiae participate in the synthesis of the iron-sulfur clusters in mitochondria (Grx5), or in signalling the iron status inside the cell for regulation of iron uptake and intracellular iron relocalization (Grx3 and Grx4). Such role in iron metabolism seems to be evolutionary conserved. Fungal cells also contain membraneassociated glutaredoxins structurally and enzymatically similar to dithiol glutaredoxins, which may act as redox regulators at the early stages of the protein secretory machinery.
\end{abstract}

Keywords: Fe-S cluster, glutaredoxin, iron homeostasis, mitochondria, oxidative stress, protein secretion, redox control 


\section{INTRODUCTION}

In the past, glutaredoxins (GRXs) have been defined as thiol oxidoreductases that employ glutathione in its reduced form (L- $\gamma$-glutamyl-L-cysteinyl-glycine, abbreviated as GSH) to regulate the redox state of protein disulfides or glutathioneprotein mixed disulfides (see reviews in [1-3]). In normal redox conditions, when the ratio between GSH and oxidized glutathione (GSSG) is high, GRXs can reduce the disulfide bonds with GSH as hydrogen donor. In these conditions, therefore, it is supposed that GRXs act as intermediates in the protein reduction process. However, in oxidant conditions, when the GSH/GSSG ratio decreases and the redox potential for the redox couple becomes less negative, GRXs can catalyze the glutathionylation of reduced sulfydryl groups in proteins [4]. Therefore, GRXs can act as modulators of protein activity by regulating the glutathionylation state of sulfydryl groups in the protein molecules [5]. In addition to GRXs, the GRX system includes glutathione reductase (which reduces GSSG to GSH) and NADPH, which acts as hydrogen donor for glutathione reductase. The role of GRXs as protein reductants became evident for the first time when it was demonstrated that an Escherichia coli GRX was able to reduce ribonucleotide reductase (RNR) [1]. Later, it was shown that GRXs also can reduce other proteins, among them 3'-phosphoadenylylsulfate reductase, a key enzyme in the conversion of inorganic sulfate into sulfur amino acids. Thus, the regulatory role of GRXs on central metabolic processes seems clear. However, during the last decade it became evident that GRXs constitute an heterogeneous protein family whose members have a large structural and functional diversity [3,6,7]. Even more, some GRXs are not active in dehydroascorbate reduction or in deglutathionylation of low molecular substrates such as $\beta$-mercaptoethanol disulfide (HEDS) [8-11], which are two standard assays for measuring the thiol oxidoreductase activity of GRXs.

Therefore, what defines a GRX? Structurally, all GRXs have a 'thioredoxinfold' structure (see below), which is formed by a four- or five-stranded $\beta$-sheet flanked by three or more $\alpha$-helices on either side of the $\beta$-sheet [12]. However, the thioredoxinfold superfamily includes other members in addition to GRXs [13]. Besides, a large number of GRX molecules have a complex structure with different types of modules fused to the GRX module with the thioredoxin-fold structure [7]. On the other hand, GRXs have a common active site motif $\mathrm{CxxC/S}$ located at a surface loop that is 
accessible to GSH. Other amino acid residues which are conserved in GRXs help to define a GSH groove that together with the active site residues, allows the interaction of a GSH molecule with a GRX monomer [3]. With these considerations, GRXs are defined on structural bases in addition to their ability to interact with GSH molecules. This leaves open the possibility for a large diversity of physiological functions and also for different molecular mechanisms of GRX action, as recent studies are revealing. In this review we will make emphasis on GRX studies in the yeast Saccharomyces cerevisiae, since this organism has allowed a diversity of experimental approaches (genetic, biochemical, cell and molecular biology) which have established the basis for further studies in multicellular organisms.

\section{GLUTAREDOXIN SUBFAMILIES}

Depending on the number of cysteine residues at the active site, GRXs are currently classified into two subfamilies: dithiol (2-Cys) and monothiol (1-Cys) GRXs. In addition, land plants contain a third type of GRXs with a CCxx active site, and three additional GRXs classes with an active site motif different from the above ones have been defined in prokaryotic and eukaryotic organisms with photosynthetic metabolism $[6,14]$. These specific GRXs from photosynthetic organisms have been less well characterized, and will not be considered here. Although we will employ the above classification for dithiol and monothiol GRXs, it should be kept in mind that it does not necessarily reflect the phylogenetic relationships among GRXs [7], and some 1-Cys GRXs may display higher homology with 2-Cys molecules than with other 1-Cys molecules [15]. Most dithiol GRXs contain a CPYC active site, although in some of them the Pro residue may be substituted by Ser or the Tyr residue by Phe. S. cerevisiae has two dithiol GRXs, which share a sequence identity of $64 \%$ : Grx1 is located at the cytosol, while Grx2 has a large cytosolic pool and a minor pool at the mitochondrial matrix and outer membrane [16,17] (Fig. 1). The existence of two Grx2 pools results from alternative translation initiation from two in-frame AUG sites [17]. The shorter isoform remains at the cytosol, while the longer one is translocated to the mitochondrial matrix. This isoform can be processed by a mitochondrial peptidase resulting in a soluble matrix form, or left unprocessed as an outer mitochondrial membrane-associated form. Human cells also contain two GRXs at different cell compartments. Grx1 is cytosolic and participates in ribonucleotide and dehydroascorbate reduction, regulation 
of transcription factors and apoptosis, while Grx2 has two isoforms (nuclear and mitochondrial) derived from the same gene by alternative transcription initiation and splicing $[18,19]$. Human Grx2 has the unusual active site motif CSYS, and the most Nterminal Cys residue is coordinated to an Fe-S cluster with the structure $[2 \mathrm{Fe}-2 \mathrm{~S}]^{2+}$ bridging two Grx2 monomers [20]. In addition to their thiol oxidoreductase activity, yeast Grx1 and Grx2 are also active as glutathione transferases (GSTs), that is, they are able to conjugate different small molecular weight organic compounds (generally of xenobiotic nature) to the sulfhydryl group of a GSH molecule [21]. These glutathionylated molecules are consequently degraded or transported into the vacuole, what constitutes a detoxification mechanism.

Monothiol GRXs were first described in S. cerevisiae, which contains three members of this subfamily: Grx3, Grx4 and Grx5 [22] (Fig. 1). They have homologues widely distributed among prokaryotic and eukaryotic organisms, and are characterized by the presence of a CGFS active site motif (reviewed in [23]). In eukaryotes, many of the monothiol GRXs contain more than one GRX module or domain in tandem, as well as domains of a different origin fused at the N-terminal region of the molecule. For instance, yeast Grx3 and Grx4 contain a thioredoxin-like domain fused to the GRX domain [24], while human PICOT protein is formed by a thioredoxin-like domain fused to two GRX domains in tandem [25]. The functional implications of such modular structure are unknown, but bioinformatics analyses have shown that fusion events have occurred repeatedly along evolution [7]. In S. cerevisiae, the thioredoxin-like domain of Grx3 is important for compartmentalization of the protein [24]. Different cell compartments contain monothiol GRXs in eukaryotic cells. Thus, S. cerevisiae Grx5 is located at the mitochondrial matrix [26], while Grx3 and Grx4 have been described at the nucleus [23] and also at the cytosol [27]. Location of human Grx5 has not been directly determined, although its functional characterization (see below) firmly supports a mitochondrial location as occurs with its yeast homologue. On the other hand, plant monothiol GRXs are present at the cytosol, chloroplasts and mitochondria, and probably also at the nucleus [6]. Members of this GRX subfamily display very low or null thiol disulfide oxidoreductase activity in standard enzyme assays for GRXs, although they may be able to deglutathionylate some glutathionylated protein substrates [8-11]. This deglutathyonulation ability occurs in prokaryotic and eukarytic monothiol GRXs. The reductant in the in vitro assays is GSH [8,9], or the couple ferredoxin plus ferredoxinthioredoxin reductase in the case of the green alga Chlamydomonas reinhardtii [10]. 
Associating the concept of monothiol (1-Cys) GRX to the CGFS-type molecules should be revised due to the description of two additional GRXs in S. cerevisiae, Grx6 and Grx7, respectively with the active site motifs CSYS and CPYS [15,28] (Fig. 1). Homologues of Grx6 and Grx7 are present in diverse fungal species, although not in other organisms, and both molecules have standard thiol disulfide oxidoreductase activity both in vitro and in vivo [15,29]. Purified Grx6 also displays GST activity, even higher than yeast Grx1 or Grx2 [30]. On the contrary, the oxidoreductase activity of Grx6 and Grx7 is considerably lower than the activity of the dithiol Grx1 and Grx2 $[15,29]$. Sequence homology analyses result in grouping Grx6 and Grx7 close to dithiol GRXs [7]. Therefore, both primary structure and enzyme activity properties indicate that Grx6 and Grx7 are closer to dithiol GRXs than to CGFS-type GRXs. On the other hand, Grx6 and Grx7 are the first GRXs characterized as integral membrane proteins of the early secretory pathway, at Golgi in the case of Grx7 and endoplasmic reticulum (ER) and Golgi in the case of Grx6 [15,28]. S. cerevisiae Grx6 and Grx7 and their fungal homologues have a complex structure consisting of an $\mathrm{N}$-terminal transmembrane domain, which is responsible for the insertion of the molecules at the membranes of the secretory vesicles, followed by a long linker domain and the GRX module (Fig. 1). The CS/PYS active site is positioned at the lumen of the vesicles [14].

Recently, another GRX has been described in S. cerevisiae, Grx8 [31] (Fig. 1). Its amino acid sequence supports a thioredoxin-fold structure for Grx8, which displays more sequence homology with dithiol GRXs than with other GRXs. The active site motif (CPDC) and the adjacent residues are more similar to thioredoxin-like proteins than to GRXs, although purified Grx8 has low but detectable thiol disulfide oxidoreductase activity in a standard GRX activity assay and can be reduced by GSH, which confirms its role as GRX [31]. Yeast mutants lacking Grx8 do not have an apparent phenotype, and therefore the physiological role of this atypical GRX remains unsolved.

\section{STRUCTURE OF GLUTAREDOXINS}

Dithiol GRXs were the first GRXs characterized at biochemical and structural levels. Consequently, the three-dimensional structure of a number of dithiol GRXs has been determined experimentally and the corresponding data are available at RCBS Protein Data Bank (PDB). They are structurally defined by the thioredoxin-fold domain 
and the conservation of a number of residues that constitute the GRX groove and/or are important for interactions with the GSH molecule [3]. The three-dimensional structure of $S$. cerevisiae Grx1 has been solved in detail [32] (PDB codes 3C1R and 3C1S respectively for the oxidized and glutathionylated forms). Compared to other dithiol GRXs for which the tertiary structure has been determined, yeast Grx1 has a number of conserved residues important for the GSH interactions (Fig. 2). These include Thr74, Val75 and Pro76, which interact with the glutamyl and cysteinyl groups of GSH, as well as Lys24, which is implicated in binding the glycine moiety of GSH [32]. The highly conserved Ile85, Gly86 and Gly87, located at the loop that connects $\beta$-sheet 4 with $\alpha$-helix 4 , are important for the adequate orientation of this $\alpha$-helix to allow forming the GSH groove. The CPYC active site is exposed to the exterior, in the loop between $\beta$-sheet 1 and $\alpha$-helix 2 . The crystal structures of oxidized and reduced $S$. cerevisiae Grx2 have also been solved very recently (PDB codes 3CTF and 3CTG respectively) [33]. Comparison of the three-dimensional structures of S. cerevisiae Grx1 and Grx2 shows that both are very similar and that the residues involved in GSH binding are highly conserved. However, Asp89 in Grx1 is substituted by Ser123 in the equivalent position in Grx2, and it has been demonstrated that this change alone may explain the higher specific activity of Grx2 compared to Grx1 in enzyme assays with HEDS as substrate [33]. These two Asp and Ser residues are implicated in the interaction with the $\gamma$-glutamyl moiety of GSH $[32,33]$.

Some dithiol GRXs such as human Grx2 (CSYC active site) or poplar GrxC1 (CGYC active site) incorporate a Fe-S cluster in the $[2 \mathrm{Fe}-2 \mathrm{~S}]^{2+}$ form that bridges two monomers to constitute an homodimeric GRX complex [20,34]. The Fe-S cluster is coordinated to each of the most N-terminal active site cysteines of the two GRX monomers and each of the cysteinyl residues of two GSH molecules. Therefore, GSH is directly involved in formation of the holo GRX dimer. Significantly, the absence of a proline residue in the active site of these two dithiol GRXs is a requisite for Fe-S binding [20,34]. In the case of human Grx2, the holodimer is enzymatically inactive, while the monomers are active. This has led to establish the hypothesis that the Fe-S cluster in the dimer would act as a redox sensor, and its disorganization upon oxidative stress would induce Grx2 activation $[35,36]$.

In spite of their sequence similarity, the structural properties of $S$. cerevisiae Grx6 and Grx7 are different. Thus, recombinant Grx6 produced in E. coli is purified 
mostly as a tetramer with two Fe-S clusters, while Grx7 is obtained as a dimer lacking Fe-S clusters [29]. In both cases, oligomerization is non-covalent. In Grx6, each Fe-S cluster is stabilized by GSH and bridges two monomers, as occurs with human Grx2 and poplar GrxC1. The Grx6 N-terminal moiety is also necessary for stabilizing the tetramers. Remarkably, the active site of Grx6 (GSYS) is similar to that of human Grx2, except for the second cysteine residue. The crystal structure of the GRX domain of Grx6 has been determined [30] (Fig. 2). It reveals an extra $\beta$-sheet at the C-terminal region, which probably is also present in Grx7 based on sequence comparison. This additional structure localizes opposite to the GSH groove, and its functional role remains to be determined. Some of the amino acid residues which interact with the GSH molecule are equivalent to those important in S. cerevisiae Grx1 or Grx2 [30] (Fig. 2).

Diverse monothiol GRXs of the CGFS type have been purified in bacterial cells. In all cases they form non-covalent homodimers interacting with a $[2 \mathrm{Fe}-2 \mathrm{~S}]^{2+}$ cluster ligated to two GSH molecules [37-39]. The cysteine residue of the active site is required for Fe-S assembly, as well as some other residues conserved in such GRXs [37]. This situation is similar to that described for some dithiol GRXs (see above). However, crystallography analysis of the dimer structure of the Escherichia coli monothiol Grx4 showed that besides the Fe-S cluster plus GSH ligand, other regions of the molecule are important for interaction between the two monomers [40]. This contrasts with dithiol human Grx2, where only the Fe-S cluster participates in monomer interactions [20]. Other important differences are observed when the monomeric [41] and dimeric [40] structures of E. coli Grx4 are compared. The monomer structure prevents the binding of the Fe-S cluster and the GSH molecule and, therefore, formation of the dimer. This together with the fact that the poplar monothiol GrxS14 can deliver Fe-S clusters in vitro to apoproteins [39], may suggest that monothiol GRXs in their dimeric form would act as Fe-S reservoirs or scaffolds to deliver them to receptor proteins (discussed below). Structural analyses of other monothiol GRXs such as S. cerevisiae Grx5 are under way [42], and the three-dimensional structure of Grx5 has been deposited in PDB (code 3GX8) (Fig. 2).

\section{CELLULAR FUNCTIONS OF DITHIOL GLUTAREDOXINS}

Studies in bacterial and mammalian cells (reviewed in [2,3]) demonstrated that dithiol GRXs act as reductants of sulfhydryl groups in enzymes which alternate the 
redox state of cysteine residues essential for their catalytic cycle, such as RNR (involved in conversion of ribonucleotides to deoxynucleotides) or 3'phosphoadenylylsulfate reductase (participating in the assimilation of sulfate to reduced sulfur compounds such as cysteine or methionine). In those processes, GRXs act in parallel to the thioredoxin (TRX) system, which is composed by TRXs, TRX reductase and NADPH, which acts as direct electron donor [1]. The relative contribution of both dithiol GRXs and TRXs seems to vary between organisms. Thus, in E. coli Grx1 appears to be the main in vivo contributor for RNR reduction [43]. On the contrary, in $S$. cerevisiae only the double trxl trx 2 mutant accumulates an oxydized form of RNR, while the grx1 grx2 mutant is not affected [44]. Therefore, in this yeast TRXs seem to be the main enzymes responsible for RNR reduction. This is in accordance with another study demonstrating that TRXs are required in $S$. cerevisiae for maintaining the deoxyribonucleotide pools during the S phase [45].

As mentioned above, dithiol GRXs are also deglutathionylating enzymes, that is, they are able to efficiently reduce mixed disulfides formed between GSH and protein sulfydryl groups in in vitro assays. Protein glutathionylation can be envisaged as a mechanism both to protect proteins from irreversible oxidation of sulfhydryl groups and to regulate protein functions [5,46,47]. For instance, human Grx1 regulates in vivo glutathionylation of specific proteins such as Ras, inhibitory kappa B kinase, actin or caspase 3 [48-51]. However, knockout mice that do not express Grx 1 are not especially sensitive to oxidants [52], what argues against a general protective role upon oxidative stress. A recent study [52] has shown that a S. cerevisiae mutant lacking both Grx1 and Grx2 does not display protein hyperglutathionylation. On the contrary, a mutant lacking the cytosolic TRXs Trx1 and Trx2 has constitutively high levels of protein glutathionylation. Therefore, at least in S. cerevisiae, it is the TRX system which seems to regulate the general glutathionylation state of cell proteins [53]. Such glutathionylation levels become increased at stationary phase [53].

Which is then the physiological role of dithiol GRXs? In the case of human Grx2, this might act as a sensor of oxidative stress through its ligated Fe-S cluster $[20,35]$. Deorganization of the cluster by oxidants would release the individual monomers in their active form. Still, the specific in vivo protein targets of human Grx2 are not known. However, most dithiol GRXs do not seem to be ligated to Fe-S clusters and therefore, the oxidative sensor role would be specific for a subset of them. In $S$. cerevisiae overexpression of Grx1 enhances protection against superoxide anion and 
hydroperoxides, while overexpression of Grx2 allows resistance to hydroperoxides [16], which suggests that both GRXs are partially specialized. Grx1 and Grx2 seem to act cooperatively with two GSTs (Gtt1 and Gtt2) in protection against hydroperoxides and toxic xenobiotics, based on studies with multiple mutants [21]. In addition, Grx1 and Grx2 detoxify the herbicide 2,4-dichlorophenoxyacetic acid [54], which generates hydroxyl radicals. Also, recent studies have shown that Grx1 and Grx2 have overlapping roles in $S$. cerevisiae in protection against toxicity by selenite $[55,56]$, which is also a generator of reactive oxygen species (ROS). Therefore, yeast Grx1 and Grx2 might target specific proteins either with an important function in protection against oxidants or which would be highly sensitive to such oxidants. Such targets remain to be characterized. Two recent studies may shed some light on the physiological role of the mitochondrial fraction of yeast Grx2. While a mutant lacking the cytosolic isoform of Grx2 does not display a different phenotype with respect to wild type cells, the mitochondrial isoform of Grx2 confers more tolerance to oxidative stress caused by high concentrations of hydrogen peroxide [57]. Related to this, in in vitro assays yeast Grx2 is specifically able to deglutathionylate the $S$. cerevisiae 1-Cys peroxiredoxin, what is an essential step to regenerate active peroxiredoxin [58]. Given the role of peroxiredoxins in peroxide detoxification, this could explain the protective role of mitochondrial Grx2 against oxidative stress generated by peroxides. It is remarkable that in the case of the mitochondrial 1-Cys peroxiredoxin, Grx2 substitutes the general role of TRXs as peroxiredoxin reductants.

Another aspect that awaits full characterization is the functional overlapping between the TRX and GRX systems. At a biochemical level, such overlapping is demonstrated by the fact that $S$. cerevisiae cells devoided of GSH reductase still are able to reduce oxidized glutathione through TRX reductase, and therefore that the TRX system can function as an alternative to maintain the GSH pool in vivo [59]. A similar situation may occur in Arabidopsis [60]. This alternative role of TRX reductase might explain why a quadruple trx $1 \operatorname{tr} x 2$ grx 1 grx 2 mutant is not viable in S. cerevisiae [61].

\section{MONOTHIOL GLUTAREDOXINS AND IRON HOMEOSTASIS}

During the last decade our knowledge on the function of monothiol GRXs of the CGFS type has increased significantly, revealing their relationship with iron metabolism and homeostasis inside the cell $[23,62]$. Initial studies were mostly made in $S$. cerevisiae 
combining genetic and biochemical approaches, and were then extended to other organisms including plants and animals. Thus, $S$. cerevisiae Grx5 was first characterized as a GRX localized at the mitochondrial matrix, which participates in the synthesis of Fe-S clusters through the ISC pathway [26]. This is one of the several pathways employed for the synthesis of these clusters in living cells. In eukaryotes it is the mechanism through which the clusters are assembled at mitochondria using an Isu1/Isu2 scaffold ( $S$. cerevisiae terminology). From the Isu1/Isu2 complex the Fe-S clusters are then transferred to mitochondrial apoproteins or exported across the mitochondrial envelope to be assembled into extramitochondrial proteins through the so called CIA machinery [63]. This export requires a number of mitochondrial envelope proteins as well as GSH. Other studies mapped the Grx5 function in the transfer of the assembled Fe-S clusters from the Isu1/Isu2 complex to the apoproteins, in combination with a Hsp70-type Ssq1 chaperone [64]. The lack of Grx5 leads to iron accumulation inside the cells, unability for respiratory growth and deffects in the activity of enzymes requiring Fe-S clusters as cofactors [26]. These phenotypes are similar to those of other ISC mutants. Using the yeast grx5 mutant model, it has been shown that bacterial, parasite, plant and vertebrate homologues are able to substitute for the function of yeast Grx5 [11,39,65,66], pointing to a functional conservation during evolution. The deficiency of Grx5 in zebrafish embryos causes hypocrhromic anemia in shiraz mutants, which can be explained because the inability to assemble Fe-S clusters, consequent activation of cytosolic Iron Regulatory Protein 1 (IRP1) and inhibition of the sysnthesis of the first enzyme of the heme synthesis pathway [67]. This phenotype was reproduced in wild-type embryos injected with grx5 antisense RNA, and expression of Grx 5 from other organisms including $S$. cerevisiae could rescue the shiraz phenotype [67]. These studies therefore revealed for the first time the relationship between the Grx5 function, the synthesis of Fe-S clusters and iron metabolism. A later study reported that a mutation in an intron of the GRX5 gene in a human patient resulted in a large decrease of GRX5 expression, deficiency in heme synthesis and sideroblastic anemia [68]. Finally, a recent work [69] has shown that Fe-S clusters can be reconstituted in vitro in recombinant human Grx5, that siRNA-mediated depletion of Grx5 in human cells inhibits maturation of Fe-S proteins and that such deficiency in erythroid cells results in accumulation of mitochondrial iron, depletion of cytosolic iron and impairment of heme synthsis, as a consequence of altered activity of IRP1. These alterations can explain the sideroblastic anemia-associated phenotype resulting from 
decreased expression of human GRX5 mRNA [68,69]. Grx5 deficiency has also been related to increased protein oxidative damage in yeast cells $[22,26]$, maybe because the abnormal iron accumulation inside the cell as a result of the disturbance of Fe-S cluster synthesis and signalling of the intracellular iron state (see below). Consequent with this, inhibition of Grx5 synthesis in human osteoblasts causes increased ROS formation and apoptosis [70].

The detailed role of Grx5 in the synthesis of the Fe-S clusters is not known. Based on the in vitro deglutathionylation activity of monothiol GRXs, it can be hypothesized that Grx5 could act maintaining in a reduced form the sulfydryl groups of acceptor proteins of Fe-S clusters at mitochondria, or alternatively it could regulate the redox state of components of the ISC machinery acting at late steps of the cluster assembly pathway. However, recently it has been shown that poplar GrxS14, a chloroplast homologue of Grx5, binds directly Fe-S clusters through the CGFS active site and is able to transfer in vitro these clusters to apoproteins such as ferredoxin [39]. This opens the possibility that Grx5 proteins in fact are intermediates in the transfer of Fe-S clusters from the scaffold complex Isu1-Isu2 (or its homologues) to the target proteins, a role that still has to be confirmed in vivo. If this were the case, then the physiologically relevant role of Grx 5 in mitochondria would not be due to its activity as thiol disulfide oxidoreductase but to its ability to form complexes with Fe-S clusters and GSH molecules. As above mentioned, structural studies indicate that the threedimensional structure of these monothiol GRXs is different in their monomeric and FeS and GSH-ligated dimeric form [40], a fact that would support the unidirectionality of the Fe-S transfer from Grx5 to the apoprotein.

Other $S$. cerevisiae CGFS-type monothiol GRXs, Grx3 and Grx4, have a nuclear and/or cytoplasmic location [23,27]. Two separate studies [71,72] demonstrated that they regulate the activity of the Aft1 transcription factor. Upon iron limitation, Aft1 activates the expression of genes involved in (i) iron assimilation through the highaffinity pathway and the siderophore-mediated pathway, and (ii) vacuolar iron transport $[73,74]$. Altogether, they are known as the iron regulon. Upon iron depletion, Aft1 localizes in the nucleus and upregulates the genes of the regulon, while in iron-replete conditions it moves to the cytosol and the iron regulon is not expressed $[75,76]$. Inhibition of mitochondrial $\mathrm{Fe}-\mathrm{S}$ cluster synthesis also causes activation of the iron regulon [77-79], and it has been proposed that a mitochondrial Fe-S protein (or another type of molecule) could act as a sensor of the intracellular iron status transferring the 
corresponding signal to Aft1 [80]. Grx3 and Grx4 form a complex with Aft1, and in the absence of the two GRXs Aft1 remains constitutively localized in the nucleus $[71,72]$. In a grx 3 grx 4 mutant, the consequent upregulation of the Aft1 regulon independently of the intracellular iron status results in higher than normal intracellular iron levels and hypersensitivity to oxidative stress [71,72]. Recently, it has been demonstrated that Grx3 and Grx4 are Fe-S proteins in vivo (in addition to the previous demonstration in vitro [37]), and that such ligated clusters are essential for the signalling role on Aft1 activity [81]. Thus, mitochondrial synthesis of Fe-S clusters would be essential for such signalling events. Fig. 3 summarizes the role of the three monothiol GRXs in the expression of the iron regulon in S. cerevisiae.

The Aft1/Grx3-4 complex contains two additional proteins, Fra1 (an aminopeptidase P-like protein) and Fra2 (a BolA homologue) [27]. In the absence of any of these two proteins there is also constitutive upregulation of the iron regulon. In vitro reconstitution experiments have demonstrated that Fra2 helps stabilizing the Aft1/Grx3-4 complex [82]. Comparative genomic analyses demonstrate that an strong cooccurrence exists in organisms between BolA-like proteins and CGFS-type GRXs $[6,83]$. This together with the studies demonstrating physical interactions between members of the two families suggest that the functional relationships between Fra and Grx3/4 homologues are evolutionary conserved.

The role of Aft1-like proteins in regulating iron homeostasis is circumscribed to some fungal species. However, the participation of homologues of Grx3 and Grx4 in the control of intracellular iron levels seems to be a more general trait. Thus, in the fission yeast Schizosaccharomyces pombe iron homeostasis is transcriptionally controlled by two factors, Fep1 (which represses expression of genes for iron acquisition) and Php4 (which represses genes for iron-requiring proteins) [84]. There is a cross-talk between both factors, since expression of the $p h p 4^{+}$gene is repressed by Fep1. Upon iron repletion, $S$. pombe Grx4 inactivates the repressor function of Php4, which is then exported to the cytosol, therefore allowing expression of iron-requiring proteins [85]. Therefore, CGFS-type GRXs seem to exert a rather general role in the regulation of transcriptional factors controlling intracellular iron homeostasis, probably acting as iron sensors. The exact mechanism through which Grx3/4 proteins transduce the signals of the iron status remain to be elucidated, but it is tempting to relate it to their properties as Fe-S proteins. 
In fact, Grx3 and Grx4 have additional functions in S. cerevisiae. In some genetic backgrounds a double mutant lacking both proteins is not viable $[81,86]$, which can not be explained based exclussively on the Aft1-mediated effects on the iron regulon. Grx4 interacts and is phosphorylated by Bud32, a protein kinase member of the EKC/KEOPS complex, otherwise involved in transcription control and telomere homeostasis [86,87]. Upon Grx3 and Grx4 depletion all iron-requiring reactions in the cytosol, mitochondria and nucleus are impaired, including the synthesis of heme, di-iron centers and Fe-S clusters [81]. Thus, Grx3 and Grx4 would be central regulators of intracellular iron trafficking in yeast cells through their Fe-S bound cluster.

The PICOT protein (also named GLRX3 or Grx3) is the homologue of Grx3 and Grx4 in human cells, and until the moment it has not been related to iron metabolism but to other regulatory processes. It was first characterized as a protein kinase $\mathrm{C}$ theta (PKC- $\theta$ )-binding protein in human $\mathrm{T}$ lymphocytes. In these cells, it inhibits PKC- $\theta-$ dependent activation of $\mathrm{JNK}$ protein kinase and the AP-1 and NF- $\kappa \mathrm{B}$ transcription factors [25]. On the other hand, PICOT is activated during cardiac hypertrophy, regulating cardiomyocyte contractility [88]. In cardiomyocytes, PICOT binds to muscle LIM protein in competition with calcineurin [89]. In this way, it disrupts calcineurinmediated activation of the NFAT factor, which itself is a regulator of cardiomyocyte contractility [89]. Nevertheless, a mouse null mutant in the PICOT gene is unable to complete embryonic development [90], indicating that PICOT may have other essential roles in addition to PKC- $\theta$ and muscle LIM protein binding in mammalian cells. All there activities depend on the cysteine residue of the GRX domain of the molecule. Structural studies with purified recombinant PICOT have shown that this is also an homodimeric Fe-S protein in which each of the two GRX domains of each monomer is ligated to a $\mathrm{Fe}-\mathrm{S}$ cluster that acts as a bridge with the other monomer [91]. The two clusters of the homodimer could act as regulators of the protein ability to interact with its targets.

\section{GLUTAREDOXINS AND THE PROTEIN SECRETORY PATHWAY}

Membranous compartments of the secretory machinery, and in particular the ER, constitute a cellular environment where ROS are generated [92,93]. This is the consequence of protein sulfhydryl oxidation and subsequent formation of disulfide 
bridges required for correct protein folding. At least two activities are required for this process: a member of the protein disulfide isomerase (PDI) family, and a FADdependent oxidase. Both form an electron transport chain that transfers electrons from the protein cysteine groups to oxygen, although ROS are also formed accidentally. As a consequence, the early secretory pathway compartment constitutes a highly oxidative environment. In S. cerevisiae, Pdil and Ero1 are respectively the isomerase and the oxidase of the ER transport chain for protein protein folding, being both essential for yeast cell growth [91]. The physiological function of Ero1 has been partially elucidated by employing conditional mutants or mutants with reduced activity [94,95]. Thus, erol1 is a thermosensitive mutant that at the restrictive temperature is unable to fold properly secretory proteins such as carboxypeptidase Y, and whose defects are partially suppressed by oxidants of sulfhydryl groups such as diamide and enhanced by reductants such an dithiothretiol. In addition, GSH is required at the ER for correct functioning of the secretory machinery [96]. These observations have led to propose a relationship between redox control and protein secretion [92].

Most secretory proteins are cotranslationally folded while transferred to the ER. However, a subgroup of proteins with a transmembrane domain at the polypeptide Cterminus (named tail-anchored proteins) are inserted post-translationally at diverse membranous organelles, among them the ER and the Golgi apparatus [97]. Compartmentalization of such proteins at the early secretory pathway has been characterized recently in $S$. cerevisiae, and it involves a GET machinery formed by a recruiting Get3 protein and a Get1/Get2 complex that inserts the tail-anchored proteins at the ER/Golgi membranes [98,99]. The GET complex is dependent on the cellular redox state, since formation of Get3 dimers (required for the activity of the protein) requires that two C-terminal cysteine residues are reduced [100]. This again establish a relationship between compartimentalization of a subset of proteins at the early secretory pathway and redox regulation of sulfhydryl groups.

In fungal cells, Grx6 and Grx7 could be such redox regulators at RE/Golgi, based on their location as integral membrane components at those compartments, although few data exist at the moment to sustain that hypothesis. Phenotypic analyses of grx6 and grx7 mutants indicate that the double mutant has increased tolerance to tunycamicin, an inhibitor of $\mathrm{N}$-glycosylation of secretory proteins [15], and that in some genetic backgrounds the grx6 mutant is hypersensitive to oxidants [28]. However, such mutants have not induced the unfolded protein response [28], a signaling cascade 
through which eukaryotic cells activate expression of diverse chaperones and heat shock proteins among others, in response to insults causing protein unfolding in the ER $[101,102]$. This indicates that Grx6 and Grx7 may play non-general but specific roles in redox regulation of protein secretion.

\section{CONCLUDING REMARKS}

Dithiol and monothiol GRXs seem to have rather different functions. Members of the first subfamily, acting as disulfide reductants, may be redox regulators of specific target proteins. Some of these dithiol GRXs, by having a slightly modified active site, have acquired the ability to bind Fe-S clusters, which could act as sensors of oxidant conditions. While Fe-S cluster ligation is the exception among dithiol GRXs, in the case of monothiol GRXs the ability to interact with Fe-S clusters seems to be general, and these GRXs may act as intermediates in the transfer of the clusters to receptor proteins. This activity seems to be important for the physiological role of monothiol GRXs in processes related to iron metabolism.

\section{ACKNOWLEDGMENTS}

Recent work of our team was supported by grants BFU2004-03167 and CSD2007-0020 (Ministerio de Ciencia e Innovación, Spain) and 2009-SGR-196 (Generalitat de Catalunya).

\section{REFERENCES}

[1] Holmgren, A. (1989) J. Biol. Chem. 254, 13963-13966.

[2] Fernandes, A.P. and Holmgren, A. (2004) Antiox. Redox Signal 6, 63-74.

[3] Lillig, C.H., Berndt, C. and Holmgren, A. (2008) Biochim. Biophys. Acta 1780, 1303-1317.

[4] Ruoppolo, M., Lundstrom-Ljung, J., Talamo, F., Pucci, P. and Marino, G. (1997) Biochemistry 36, 12259-12267.

[5] Ghezzi, P. (2005) Biochem. Soc. Trans. 33, 1378-1397. 
[6] Couturier, J., Jacquot, J.P. and Rouhier, N. (2009) Cell. Mol. Life Sci. 66, 25392557.

[7] Alves, R., Vilaprinyó, E., Sorribas, A. and Herrero, E. (2009) BMC Evol Biol 9, 66.

[8] Tamarit, J., Bellí, G., Cabiscol, E., Herrero, E. and Ros, J. (2003) J. Biol. Chem. $278,25745-25751$.

[9] Fernandes, A.P., Fladvad, M., Berndt, C., Andersen, C., Lillig, C.H.,, Neubauer, P., Sunnerhagen, M., Holmgren, A. and Vlamis-Gardikas, A. (2005) J. Biol. Chem. 280, 24544-24552.

[10] Zaffagnini, M., Michelet, L., Massot, V., Trost, P. and Lemaire, S.D. (2008) J. Biol. Chem. 283, 8868-8876.

[11] Filser, M., Comini, M.A., Molina-Navarro, M.M., Dirdjaja, N., Hererro, E. and Krauth-Siegel, L. (2008) Biol. Chem. 389, 21-32.

[12] Martin, J.L. (1995) Structure 3, 245-250.

[13] Atkinson, H.L. and Babbitt, P.C. (2009) PLoS Comput. Biol. 5, e1000541.

[14] Rouhier, N. (2010) New Phytol. 186, 365-372.

[15] Izquierdo, A., Casas, C., Mühlenhoff, U., Lillig, C.H. and Herrero, E. (2008) Eukaryot. Cell 7, 1415-1426.

[16] Luikenhuis, S., Perrone, G., Dawes, I. and Grant, C.M. (1998) Mol. Biol. Cell 9, 1081-1091.

[17] Porras, P., Padilla, C., Krayl, M., Voos, W. and Bárcena, J.A. (2006) J. Biol. Chem. 281, 16551-16562.

[18] Lundberg, M., Johanson, C., Chandra, J., Enoksson, M., Jacobsson, G., Ljung, J., Johansson, M. and Holmgren, A. (2001) J. Biol. Chem. 276, 26269-26275.

[19] Gladyshev, V. N., Liu, A., Novoselov, S. V., Krysan, K., Sun, Q. A., Kryukov, V. M., Kryukov, G. V. and Lou, F. (2001) J. Biol. Chem. 276, 30374-30380.

[20] Lillig, C. H., Berndt, C., Vergnolle, O., Lçnn, M. E., Hudemann, C., Bill, E. and Holmgren, A. (2005) Proc. Natl. Acad. Sci. USA 102, 8168-8173.

[21] Collinson, E.J. and Grant, C.M. (2002) J. Biol. Chem. 278, 22492-22497.

[22] Rodríguez-Manzaneque, M.T., Ros, J., Cabiscol, E., Sorribas, A. and Herrero, E. (1999) Mol. Cell. Biol. 19, 8180-8190.

[23] Herrero, E. and de la Torre-Ruiz, M.A. (2007) Cell. Mol. Life Sci. 64, 15181530. 
[24] Molina, M.M., Bellí, G., de la Torre, M.A., Rodríguez-Manzaneque, M.T. and Herrero, E. (2004) J. Biol. Chem. 279, 51923-51930.

[25] Witte, S., Villalba, M., Bi, K., Liu, Y., Isakov, N. and Altman, A. (2000) J. Biol. Chem. 275, 1902-1909.

[26] Rodríguez-Manzaneque, M.T., Tamarit, J., Bellí, G., Ros, J. and Herrero, E. (2002) Mol. Biol. Cell 13, 1109-1121.

[27] Kumánovics, A., Chen, O.S., Li, L., Bagley, D., Adkins, E.M., Lin, H., Dingra, N.N., Outten, C.E., Keller, G., Winge, D., Ward, D.M. and Kaplan, J. (2008) J. Biol. Chem. 283, 10276-10286.

[28] Mesecke, N., Spang, A., Deponte, M. and Herrmann, J.M. (2008) Mol. Biol. Cell 19, 2673-2680.

[29] Mesecke, N., Mittler, S., Eckers, E., Herrmann, J.M. and Deponte, M. (2008) Biochemistry 47, 1452-1463.

[30] Luo, M., Jiang, Y.L., Ma, X.X., Tang, Y.J., He, Y.X., Yu, J., Zhang, R.G., Chen, Y. and Zhou, C.Z. (2010) J. Mol. Biol. 398, 614-622.

[31] Eckers, E., Bien, M., Stroobant, V., Herrmann, J.M. and Deponte, M. (2009) Biochemistry 48, 1410-1423.

[32] Yu, J., Zhang, N.N., Yin, P.D., Cui, P.X. and Zhou, C.Z. (2008) Proteins 72, 1077-1083.

[33] Li, W.F., Yu, J., Ma, X.X., Teng, Y.B., Luo, M., Tang, Y.J. and Zhou, C.Z. (2010) Biochim. Biophys. Acta 1804, 1542-1547.

[34] Rouhier, N., Unno, H., Bandyopadhyay, S., Masip, L., Kim, S.K., Hirasawa, M., Gualberto, j.M., Lattard, V., Kusunoli, M., Knaff, D.B., Georgiou, G., Hase, T., Johnson, M.K. and Jacquot, J.P. (2007) Proc. Natl. Acad. Sci. USA 104, 73797384.

[35] Johansson, C., Kavanagh, K.L., Gileadi, O. and Oppermann, U. (2007) J. Biol. Chem. 282, 3077-3082.

[36] Berndt, C., Hudemann, C., Hanschmann, E.M., Axelsson, R., Holmgren, A. and Lillig, C.H. (2007) Antioxid. Redox Signal 9, 151-157.

[37] Picciocchi, A., Saguez, C., Boussac, A., Cassier-Chauvat, C. and Chauvat, F. (2007) Biochemistry 46, 15018-15026.

[38] Comini, M., Retting, J., Dirdjaja, N., Hanschmann, E., Berndt, C. and KrauthSiegel, R. (2008) J. Biol. Chem. 283, 27785-27798. 
[39] Bandyopadhyay, S., Gama, F., Molina-Navarro, M., Gualberto, J., Claxton, R., Naik, S., Huynh, B., Herrero, E., Jacquot, J.P., Johnson, M. and Rouhier, N. (2008) EMBO J. 27, 1122-1133.

[40] Iwema, T., Picciocchi, A., Traore, D.A.K., Ferrer, J.L., Chauvat, F. and Jacquamet, L. (2009) Biochemistry 48, 6041-6043.

[41] Fladvad, M., Bellanda, M., Fernandes, A., Mammi, S., Vlamis-Gardikas, A., Holmgren, A. and Sunnerhagen, M. (2005) J. Biol. Chem. 280, 24553-24561.

[42] Wang, Y., He, Y.X., Yu, J. and Zhou, C.Z. (2009) Acta Crystallogr. Sect. F: Struct. Biol. Cryst. Commun. 65, 651-653.

[43] Potamitou, A., Holmgren, A. and Vlamis-Gardikas, A. (2002) J. Biol. Chem. 277, 18561-18567.

[44] Camier, S., Ma, E., Leroy, C., Pruvost, A., Toledano, M. and Marsolier-Kergoat, M.C. (2007) Free Rad. Biol. Med. 42, 1008-1016.

[45] Koc, A., Mathews, C.K., Wheeler, L.J., Gross, M.K. and Merrill, G.F. (2006) J. Biol. Chem. 281, 15058-15063.

[46] Cotgreave, I.A. and Gerdes, R.G. (1999) Biochem. Biophys. Res. Commun. 242, $1-9$.

[47] Biswas, S., Chida, A.S and Rahman, I. (2006) Biochem. Pharmacol. 71, 551564.

[48] Adachi, T., Pimentel, D.R., Heibeck, T., Hou, X., Lee, Y.J., Jiang, B., Ido, Y. and Cohen, R.A. (2004) J. Biol. Chem. 279, 29857-29862.

[49] Reynaert, N.L., van der Vliet, A., Guala, A.S., McGovern, T., Hristova, M., Pantano, C., Heintz, N.H., Heim, J., Ho, Y.S., Matthews, D.E., Wouters, E.F. and Janssen-Heininger, Y.M. (2006) Proc. Natl. Acad. Sci. USA 103, 1308613091

[50] Wang, J.E., Tekle, E., Oubrahim, H., Mieyal, J.J., Stadtman, E.R. and Chock, P.B. (2003) Proc. Natl. Acad. Sci. USA 100, 5103-5106.

[51] Pan, S. and Berk, B.C. (2007) Circ. Res. 100, 213-219.

[52] Ho, Y.S., Xiong, Y., Ho, D.S., Gao, J., Chua, B.H., Pai, H. and Mieyal, J.J. (2007) Free Rad. Biol. Med. 43, 1299-1312.

[53] Greetham, D., Vickerstaff, J., Shenton, D., Perrone, G.G., Dawes, I.W. and Grant, C.M. (2010) BMC Biochem. 11, 3.

[54] Teixeira, M.C., Telo, J.P., Duarte, N.F. and Sá-Correia, I. (2004) Biochem. Biophys. Res. Commun. 324, 1101-1107. 
[55] Lewinska, A. and Bartosz, G. (2008) Fungal Genet. Biol. 45, 1182-1187.

[56] Izquierdo, A., Casas, C. and Herrero, E. (2010) Microbiology

[57] Porras, P., McDonagh, B., Pedrajas, J.R., Bárcena, J.A. and Padilla, C.A. (2010) Biochim. Biophys. Acta 1804, 839-845.

[58] Pedrajas, J.R., Padilla, C.a., McDonagh, B. and Bárcena, J.A. (2010) Antiox. Redox Signal

[59] Tan, S.X., Greetham, D., Raeth, S., Grant, C.M., Dawes, I.W. and Perrone, G.G. (2010) J. Biol. Chem. 285, 6118-6126.

[60] Marty, L., Siala, W., Schwarzländer, M., Fricker, M.D., Wirtz, M., Sweetlove, L.J., Meyer, A.J., Reichheld, J.P. and Hell, R. (2009) Proc. Natl. Acad. Sci. USA 106, 9109-9114.

[61] Draculic, T., Dawes, I.W. and Grant, C.M. (2000) Mol. Microbiol. 36, 11671174.

[62] Rouhier, N., Couturier, J., Johnson, M.K. and Jacquot, J.P. (2010) Trends Biochem. Sci. 35, 43-52.

[63] Lill, R. and Mühlenhoff, U. (2008) Annu. Rev. Biochem. 77, 669-700.

[64] Mühlenhoff, U., Gerber, J., Richhardt, N. and Lill, R. (2003) EMBO J. 22, 48154825.

[65] Molina-Navarro, M.M., Casas, C., Piedrafita, L., Bellí, G. and Herrero, E. (2006) FEBS Lett. 580, 2273-2280.

[66] Cheng, N.H., Liu, J.Z., Brock, A., Nelson, R.S. and Hirschi, K.D. (2006) J. Biol. Chem. 281, 26280-26288

[67] Wingert, R.A., Galloway, J.L., Barut, B., Foott, H., Fraenkel, P., Axe, J.L., Weber, G.J., Dooley, K., Davidson, A.J., Schmidt, B., Paw, B.H., Shaw, G.C., Kingsley, P., Palis, J., Schubert, H., Chen, O., Kaplan, J., the Tubingen 2000 Screen Consortium and Zon, L.I.. (2005) Nature 436, 1035-1039.

[68] Camaschella, C., Campanella, A., De Falco, L., Boschetto, L., Merlini, R., Silvestri, L., Levi, S. and Iolascon, A. (2007) Blood 110, 1353-1358.

[69] Ye, H., Jeong, S.Y., Ghosh, M.C., Kovtunovych, G., Silvestri, L., Ortillo, D., Uchida, N., Tisdale, J., Camaschella, C. and Rouault, T.A. (2010) J. Clin. Invest. 120, 1749-1761.

[70] Linares, G.R., Xing, W, Govoni, K.E., Chen, S.T. and Mohan, S. (2009) Bone $44,795-804$. 
[71] Ojeda, L., Keller, G., Mühlenhoff, U., Rutherford, J.C., Lill, R., and Winge, D. (2006) J. Biol. Chem. 281, 17661-17669.

[72] Pujol-Carrión, N., Bellí, G., Herrero, E., Nogués, A., and de la Torre-Ruiz, M.A. (2006) J. Cell Sci. 119, 4554-4564.

[73] Philpott, C.C. and Protchenko, O. (2008) Eukaryot. Cell 7, 20-27.

[74] Kaplan, C.D. and Kaplan, J. (2009) Chem Rev. 109, 4536-4552.

[75] Yamaguchi-Iwai, Y., Ueta, R., Fukunaka, A., and Sasaki, R. (2002) J. Biol. Chem. 277, 18914-18918.

[76] Ueta, R., Fujiwara, N., Iwai, K. and Yamaguchi-Iwai, Y. (2007) Mol. Biol. Cell 18, 2980-2990.

[77] Foury, F. and Talibi, D. (2001) J. Biol. Chem. 276, 7762-7768.

[78] Bellí, G., Molina, M.M., García-Martínez, J., Pérez-Ortín, J.E. and Herrero, E. (2004) J. Biol. Chem. 279, 12386-12395.

[79] Hausmann, A., Samans, B., Lill, R., and Mühlenhoff, U. (2008) J. Biol. Chem. $283,8318-8330$

[80] Rutherford, J.C., Ojeda, L., Balk, J., Mühlenhoff, U., Lill, R., and Winge, D.R. (2005) J. Biol. Chem. 280, 10135-10140

[81] Mühlenhoff, U., Molik, S., Godoy, J.R., Uzarska, M.A., Richter, N., Seubert, A., Zhang, Y., Stubbe, J., Pierrel, F., Herrero, E., Lillig, C.H., and Lill, R. (2010) Cell Metab.

[82] Li, H., Mapolelo, D.T., Dingra, N.N., Naik, S.G., Lees, N.S., Hoffman, B.M., Riggs-Gelasco, P.J., Huynh, B.H., Johnson, M.K. and Outten, C.E. (2009) Biochemistry 48, 9569-9581.

[83] Huynen, M.A., Spronk, C.A, Gabaldón, T. and Snel, B. (2005) FEBS Lett. 579, 591-596.

[84] Bird, A.J. (2008) Adv. Microb. Physiol. 53, 231-267.

[85] Mercier, A. and Labbé, S. (2009) J. Biol. Chem. 284, 249-262.

[86] Peggion, C., Lopreiato, R., Casanova, E., Ruzzene, M., Facchin, S., Pinna, L.A., Carignani, G. and Sartori, G. (2008) FEBS J. 275, 5919-5933.

[87] Lopreiato, R., Facchin, S., Sartori, g., Arrigoni, G., Casonato, S., Ruzzene, M., Pinna, L.A. and Carignani, G. (2004) Biochem. J. 377, 395-405.

[88] Jeong, D., Cha, H., Kim, E., Kang, M., Yang, D.K., Kim, J.M., Yoon, P.O., Oh, J.G., Bernecker, O.Y., Sakata, S., Le, T.T., Cui, L., Lee, Y.H., Kim do, H., Woo, S.H., Liao, R., Hajjar, R.J. and Park, W.J. (2006) Circ. Res. 99, 307-314. 
[89] Jeong, D., Kim, J.M., Cha, H., Oh, J.G., Park, J., Yun, S.H., Ju, E.S., Jeon, E.S., Hajjar, R.J. and Park, W.J. (2008) Circ. Res. 102, 711-719.

[90] Cha, H., Kim, J.M., Oh, J.G., Jeong, M.H., Park, C.S., Park, J., Jeong, H.J., Park, B.K., Lee, Y.H., Jeong, D., Yang, D.K., Bernecker, O.Y., Kim do, H., Hajjar, R.J. and Park, W.J. (2008) J. Mol. Cell. Cardiol. 45, 796-803.

[91] Haunhorst, P., Berndt, C., Eitner, S., Godoy, J.R. and Lillig, C.H. (2010) Biochem. Biophys. Res. Commun. 394, 322-326.

[92] Tu, B.P. and Weissman, J.S. (2004) J. Cell Biol. 164, 341-346.

[93] Chakravarthi, S., Jessop, C.E. and Bulleid, N.J. (2006) EMBO Rep. 7, 271-275.

[94] Frand, A.R. and Kaiser, C.A. (1998) Mol. Cell 1, 161-170.

[95] Pollard, M.G., Travers, K.J. and Weissman, J.S. (1998) Mol. Cell 1, 171-182.

[96] Cuozzo, J.W. and Kaiser, C.A. (1999) Nat. Cell Biol. 1, 130-135.

[97] Borgese, N., Brambillasca, S. and Colombo, S. (2007) Curr. Op. Cell Biol. 19, 368-375.

[98] Schuldiner, M., Collins, S.R., Thompson, N.J., Denic, V., Bhamidipati, A., Punna,T., Ihmels, J., Andrews, B., Boone, C., Greenblatt, J.F., Weissman, J.S. and Krogan, N.J. (2005) Cell 123, 507-519.

[99] Schuldiner, M., Metz, J., Schmid, V., Denic, V., Rawalska, M., Schmitt, H.D., Schwappach, B. and Weissman, J.S. (2008) 134, 634-645.

[100] Metz, J., Wachter, A., Schmid, B., Bujnicki, J.M. and Schwappach, B. (2006) J. Biol. Chem. 281, 410-417.

[101] Travers, K.J., Patil, C.K., Wodicka, L., Lockhart, D.J., Weissman, J.S. and Walter, P. (2000) Cell 101, 249-258

[102] Bernales, S., Papa, F.R. and Walter, P. (2006) Annu. Rev. Cell Dev. Biol. 22, 487-508.

\section{FIGURE LEGENDS}

Fig. (1). Domain structure of $S$. cerevisiae GRXs.

The position of the most $\mathrm{N}$-terminal cysteine of the active site is indicated in each case, as well as the cleavage site of the mitochondrial targeting sequence (MTS) and the residues limiting the transmembrane (TM) domain.

Fig. (2). Three-dimensional structures of $S$. cerevisiae GRXs. 
The structures of glutathionylated Grx1 (PDB code 3C1S), monomeric Grx6 (3L4N) and monomeric Grx5 (3GX8) are represented using Swiss-PdbViewer 4.0.1. The active site cysteine residues, as well as other residues important for interaction with GSH, are indicated. Secondary structure domains are also labelled. See Ref. 32 and 30 for more details on the structures of Grx 1 and Grx6 respectively. Note that only the structure of the GRX domain of Grx6 was determined. The residues important in Grx5 for interaction with GSH have been extrapolated from data on E. coli Grx4 in Ref. 40.

Fig. (3). Role of monothiol GRXs in the expression of the iron regulon in $S$. cerevisiae.

Under iron replete conditions the mitochondrial ISC machinery, including Grx5, is functional for the synthesis of Fe-S clusters (here depicted by blue and yellow circles). An uncharacterized intermediate containing an Fe-S cluster acts as a signal that is transmitted to the cytosol. Grx3/4 with its Fe/S cluster ligand forms a complex with Fra1/2 and with Aft1, which is retained at the cytosol. In these conditions, the iron regulon is not expressed. Under iron deprivation, the ISC machinery does not function optimally and the Fe-S mitochondrial signal is not exported to the cytosol. Grx3/4 still forms a complex with Fra1/2 but not with Aft1, which is internalized into the nucleus, allowing expression of the iron regulon. 


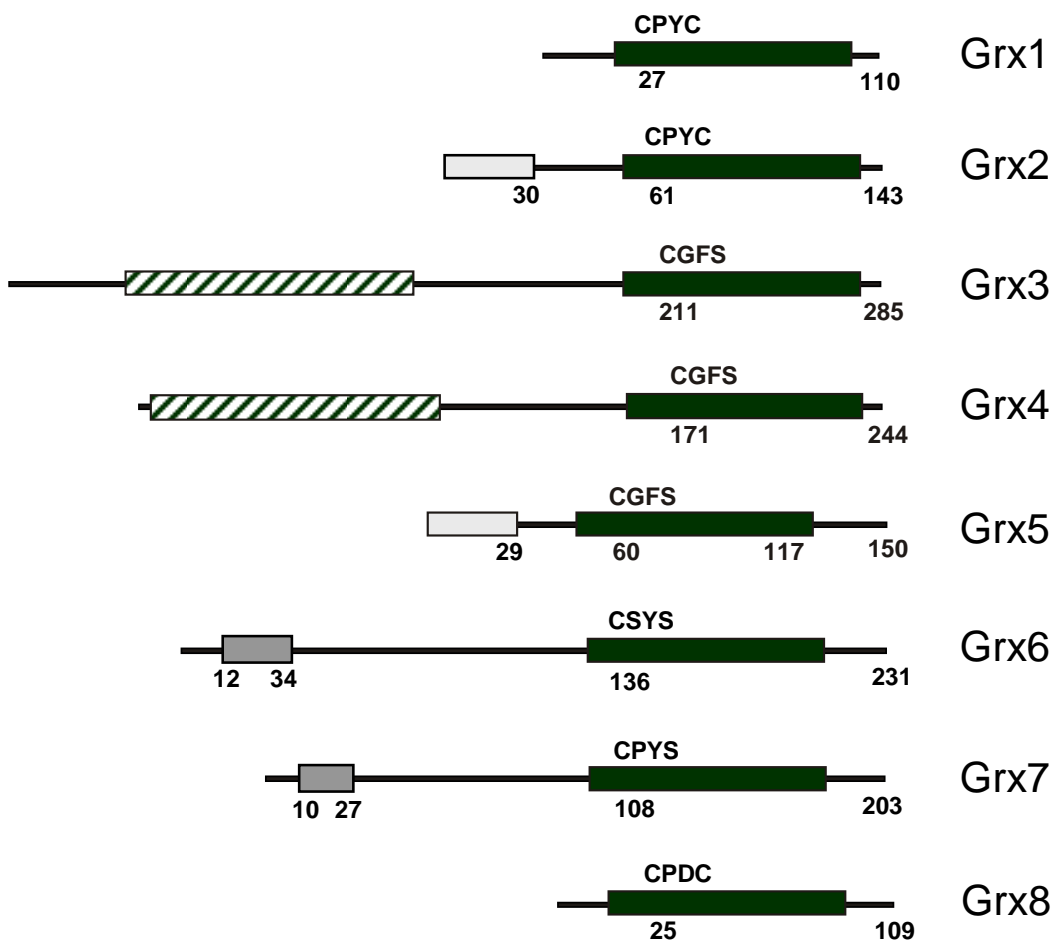

GRX domain

MTS

WIIZ TRX-like domain

TM domain 


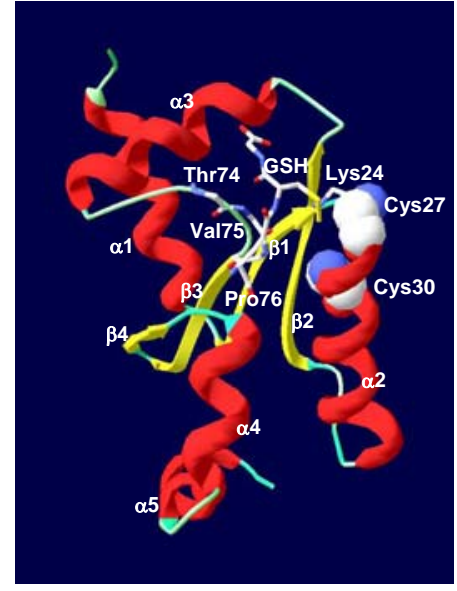

Grx1

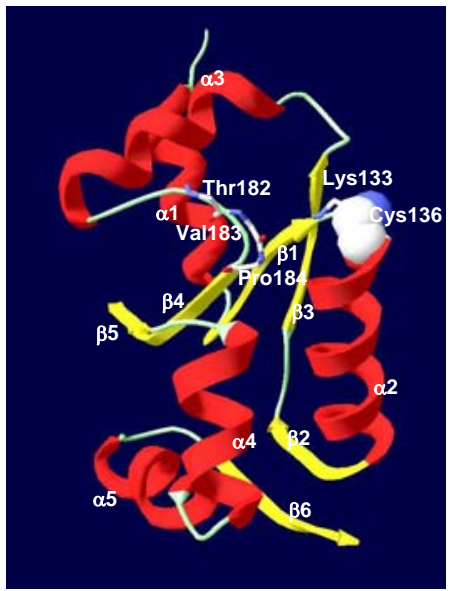

Grx6



Grx5 
Iron availability

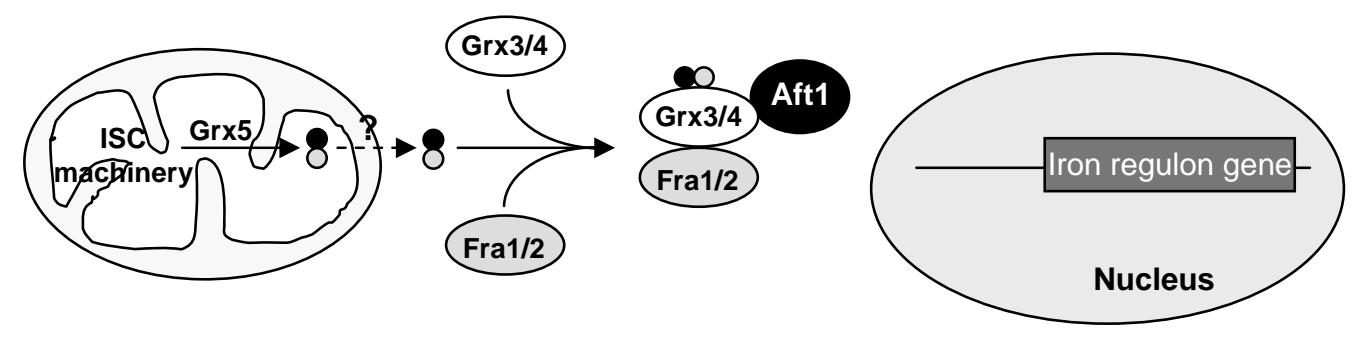

Iron depletion
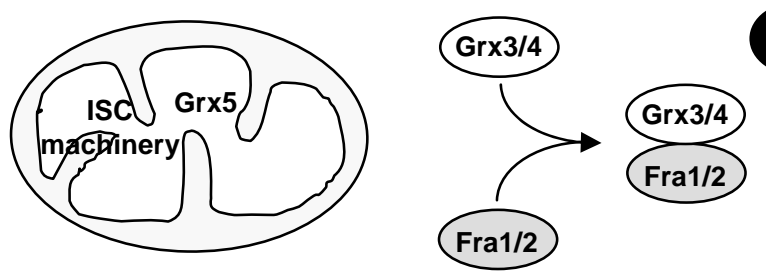

Aft1

Aft1

Iron regulon gene

Nucleus 